\title{
The Characterization of Different Flavodoxin Reductase-Flavodoxin (FNR-Fld) Interactions Reveals an Efficient FNR-Fld Redox Pair and Identifies a Novel FNR Subclass
}

\author{
Ingvild Gudim, ${ }^{* \dagger}{ }^{\dagger}$ Marta Hammerstad, ${ }^{\dagger}$ Marie Lofstad, ${ }^{\dagger}$ and Hans-Petter Hersleth*, ${ }^{\dagger, \ddagger(0)}$ \\ ${ }^{\dagger}$ Department of Biosciences, Section for Biochemistry and Molecular Biology, University of Oslo, Oslo 0316, Norway \\ Department of Chemistry, Section for Chemical Life Sciences, University of Oslo, Oslo 0316, Norway
}

Supporting Information

ABSTRACT: Flavodoxins (Flds) are small, bacterial proteins that transfer electrons to various redox enzymes. Flavodoxins are reduced by ferredoxin/flavodoxin $\mathrm{NADP}^{+}$oxidoreductases (FNRs), but little is known of the FNR-Fld interaction. Here, we compare the interactions of two flavodoxins (Fld1-2), one flavodoxin-like protein (NrdI), and three different thioredoxin reductase (TrxR)-like FNRs (FNR1-3), all from Bacillus cereus. Steady-state kinetics shows that the FNR2-Fld2 electron transfer pair is particularly efficient, and redox potential measurements also indicate that this is the most favorable electron donor/acceptor pair. Furthermore, crystal structures of FNR1 and FNR2 show that the proteins have crystallized in different conformations, a closed and an open conformation, respectively.

We suggest that a large-scale conformational rearrangement takes place during the FNR catalytic cycle to allow for the binding and reduction of the Fld and, subsequently, the re-reduction of the FNR by NADPH. Finally, inspection of the residues surrounding the FAD cofactor in the FNR active site shows that a key isoalloxazine ring-stacking residue is different in FNR1 and FNR2, which could explain the large difference in catalytic efficiency between the two FNRs. To date, all of the characterized TrxR-like FNRs have a residue with aromatic character stacking against the FAD isoalloxazine ring, and this has been thought to be a conserved feature of this class of FNRs. FNR1, however, has a valine in this position. Bioinformatic analysis shows that the TrxR-like FNRs can actually be divided into two groups, one group where the FAD-stacking residue has aromatic character and another group where it is valine.
$\mathrm{F}$ lavodoxins (Flds) are small electron-shuttling proteins that are widely distributed in bacteria. ${ }^{1}$ They serve as electron donors to several bacterial redox enzymes, for example, the ribonucleotide reductase system, nitric oxide synthase, biotin synthase, pyruvate formate-lyase, and cobalamin-dependent methionine synthase. ${ }^{2-7}$ Even if flavodoxins are not found in higher eukaryotes, domains homologous to flavodoxins are found in multidomain eukaryotic proteins, such as nitric oxide synthase and cytochrome $\mathrm{P} 450$ reductase. ${ }^{1,8}$

Flavodoxins are reduced by ferredoxin/flavodoxin $\mathrm{NADP}^{+}$ oxidoreductases (FNRs), which catalyze the redox reaction between ferredoxin or flavodoxin and NADPH. FNRs can be separated into two main families, plant-type FNRs and glutathione reductase (GR)-like FNRs, which are structurally and phylogenetically unrelated. ${ }^{9}$

Multiple studies have investigated flavodoxin interactions in the various flavodoxin-specific redox pathways. In contrast, the interaction between Flds and FNRs is not very well characterized. Some Fld-FNR interaction studies have been carried out, but these have mostly investigated FNR-Fld interactions using FNRs from the plant-type FNR superfamily and not FNRs from the GR-like FNR family. ${ }^{8,10}$ Furthermore, many organisms have several flavodoxins and flavodoxin reductases, and it has been shown that the reduction rates of various redox enzymes by the FNR-Fld system can depend on which of the endogenous flavodoxins or flavodoxin reductases is employed. ${ }^{3,11}$ Yet, other redox enzymes are reduced with a similar efficiency regardless of the flavodoxin used. ${ }^{12}$ Given that the FNR-Fld system can serve as an electron source to a number of biotechnologically relevant enzymes, ${ }^{12,13}$ it is important to recognize the reasons behind the possible differences in enzyme activity that can arise merely from which FNR-Fld system is used to deliver electrons. Also, since FNR and Fld domains are fused into multidomain enzymes in higher eukaryotes, characterizing the electron transfer interaction between these proteins in bacteria can help understand the catalytic mechanism of the more complex eukaryotic multidomain proteins.

To gain insight into the specificity and selectivity of the FNR-Fld interaction, we attempted to compare and contrast

Received: June 21, 2018

Revised: August 8, 2018

Published: August 24, 2018 
Scheme 1. B. cereus Flavoprotein Redox Network ${ }^{a}$

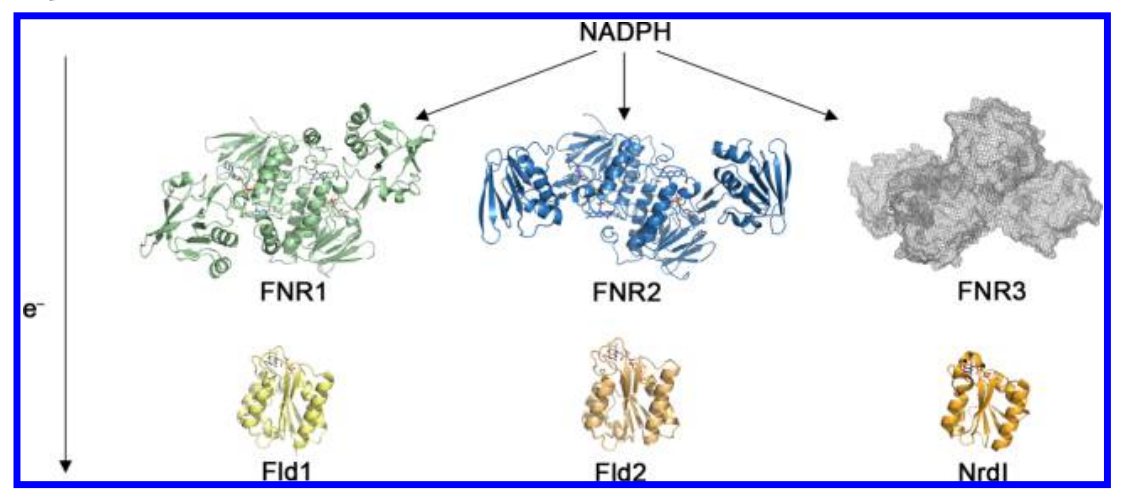

${ }^{a}$ FNR1-3 catalyzes the reaction between NADPH and Fld1, Fld2, and NrdI.

the interactions of the different FNRs and Flds from the Firmicute Bacillus cereus (Scheme 1). B. cereus has two flavodoxins, Fld1 and Fld2, and one flavodoxin-like protein, NrdI. There are three B. cereus FNRs, FNR1-3, which belong to the dimeric thioredoxin reductase (TrxR)-like subfamily of the GR-like FNRs. As the name implies, the TrxR-like FNRs share sequence and structural homology with TrxRs; however, they lack their essential CXXC catalytic motif. In characterizing this redox network, we wanted to find out whether the reduction rates of the different FNR-Fld pairs would differ and, if so, what factors contribute to the dissimilarity in reduction rates. We have shown in a previous study how the B. cereus FNRs reduce the flavodoxin-like protein NrdI at different rates, ${ }^{11}$ and here we are extending this analysis to include reduction kinetics studies of all 6 FNR-Fld pairs, as well as redox potential measurements of the FNRs, Flds, and NrdI. We also present crystal structures of two of the FNRs, FNR1 and FNR2, and one of the Flds, Fld2, supplementing the already published structures of B. cereus $\mathrm{Fld}^{14}$ and NrdI. ${ }^{15}$

\section{METHODS}

Protein Expression, Purification, and Crystallization. The expression and purification of Fld1 is described in reference 14 , and this procedure was carried out with minor changes. In brief, after protein expression, lysis, and DNA removal, contaminating proteins were precipitated by the addition of solid ammonium sulfate to a concentration of $0.25 \mathrm{~g} / \mathrm{mL}$, before the Fld 1 protein was precipitated by adding ammonium sulfate to a final concentration of $0.5 \mathrm{~g} / \mathrm{mL}$. After desalting on a column containing Sephadex G-25 Fine material (GE Healthcare) using purification buffer $(50 \mathrm{mM}$ Tris- $\mathrm{HCl} \mathrm{pH} 7.5)$, the protein was applied on a column containing HP Q anion-exchange material (GE Healthcare) and eluted using a gradient of $200-500 \mathrm{mM}$ $\mathrm{KCl}$ in the purification buffer over 20 column volumes. After concentrating the protein, it was applied on a column containing Superdex 200 size exclusion media (GE Healthcare) equilibrated with $50 \mathrm{mM}$ HEPES $\mathrm{pH} 7.5,100 \mathrm{mM} \mathrm{KCl}$ and eluted with the same buffer. The protein was concentrated, aliquoted, and flash-frozen in liquid $\mathrm{N}_{2}$, before being stored at $-80{ }^{\circ} \mathrm{C}$.

The cloning, transformation, expression, and purification protocol of the Fld2 protein used for crystallization is similar to that of Fld1. ${ }^{14}$ Here, the gene-specific forward and reverse primers were 5'-GGAATTCCATATGTTAGAGGGGGATGCCAAAGTGG-3' (F) and 5'-GCCGGATCCTTAGAACTTCTCAGCAAATGCAATTGC-3' (R). Changes in the purification protocol are as follows: after desalting in purification buffer (50 mM Tris- $\mathrm{HCl}$ pH 7.8, $100 \mathrm{mM} \mathrm{NaCl}$, $5 \%$ glycerol), the protein was applied on a HiTrap QHP anionexchange column (GE Healthcare) and eluted using a stepwise gradient of $300-500 \mathrm{mM} \mathrm{KCl}$ in the purification buffer, before gel filtration on a Superdex 75 10/300 column (GE Healthcare) equilibrated in $50 \mathrm{mM}$ Tris- $\mathrm{HCl} \mathrm{pH} 7.8,100 \mathrm{mM} \mathrm{NaCl}, 5 \%$ glycerol.

Due to low protein yields using this gene, a new, codonoptimized gene was utilized to produce the Fld2 protein used for reduction kinetics and redox potential measurements. pET$22 \mathrm{~b}(+)$ plasmid containing the gene BC3541 (B. cereus ATCC 14579, GenScript) and restriction sites NdeI-BamHI without any tags was transformed into competent BL21 (DE3) cells (Novagen). The expression, lysis, and purification were carried out almost as described for the Fld1. In brief, the cells containing Fld 2 were lysed using either an X-press ${ }^{16}$ or by sonication ( $80 \%$ amplitude, $20 \mathrm{~s}$ on, $40 \mathrm{~s}$ off). After removal of cell debris and DNA, contaminating proteins were precipitated by adding solid ammonium sulfate to a concentration of $0.3 \mathrm{~g} / \mathrm{mL}$, before the Fld 2 protein was precipitated by the addition of $0.5 \mathrm{~g} / \mathrm{mL}$ ammonium sulfate in total. After desalting on a column containing Sephadex G-25 Fine material (GE Healthcare) using purification buffer (50 mM Tris- $\mathrm{HCl}, \mathrm{pH} 7.5)$, the protein was applied on a column containing $\mathrm{HP} \mathrm{Q}$ anionexchange material (GE Healthcare) and eluted using a gradient of $300-600 \mathrm{mM} \mathrm{KCl}$ in the purification buffer over 20 column volumes. After concentrating the protein, it was applied on a column containing Superdex 200 material (GE Healthcare) or a Superdex 75 10/300 Increase column (GE Healthcare) equilibrated with $50 \mathrm{mM}$ HEPES $\mathrm{pH} 7.5,100 \mathrm{mM} \mathrm{KCl}$ and eluted with the same buffer. The protein was concentrated, aliquoted, and flash-frozen in liquid $\mathrm{N}_{2}$, before being stored at $-80{ }^{\circ} \mathrm{C}$.

The concentrations of both Fld 1 and Fld 2 were estimated using a molar extinction coefficient of $10 \mathrm{mM}^{-1} \mathrm{~cm}^{-1}$ at $450 \mathrm{~nm}^{3}$.

Protein expression and purification of the FNRs were carried out using the same procedure as described in the work by Lofstad et al. ${ }^{11}$

The crystallization conditions of FNR1 are reported by Skråmo and co-workers. ${ }^{17}$ For FNR2, NADP $^{+}$(Sigma) and FNR2 were mixed so that the final $\mathrm{NADP}^{+}$concentration was $5 \mathrm{mM}$ and the FNR2 concentration $16 \mathrm{mg} / \mathrm{mL}$. The FNR2/ $\mathrm{NADP}^{+}$solution was then mixed in a 1:1 ratio with the reservoir solution, with a total drop volume of $400 \mathrm{~nL}$. FNR2 crystallized in the $\mathrm{C} 1$ condition ( $0.1 \mathrm{M}$ sodium chloride, $0.02 \mathrm{M}$ Tris $\mathrm{pH} 7.0,7.7 \% \mathrm{w} / \mathrm{v}$ PEG 4000) in the Memgold screen (Molecular Dimensions). FNR2 crystals were fished directly 
from the screen, cryo-protected in crystallization solution containing 30\% glycerol, and flash-frozen in liquid $\mathrm{N}_{2}$. Crystal hits for Fld2 were obtained with the JCSG+ screen (Qiagen), and the final crystallization solution contained $0.2 \mathrm{M}$ calcium acetate, $40 \%(\mathrm{v} / \mathrm{v}) \mathrm{PEG} 300$, and $0.1 \mathrm{M}$ sodium cacodylate $\mathrm{pH}$ 6.5. The Fld 2 crystals were cryo-protected in mother liquor containing $30 \%$ glycerol and flash-frozen in liquid $\mathrm{N}_{2}$.

Crystal Data Collection and Processing. Diffraction data were collected at the SLS in Villigen, Switzerland, on beamline X10SA (Fld2) and at the ESRF in Grenoble, France, on beamline ID30A-3 (FNR2). Data collection of FNR1 crystals and subsequent data processing are described by Skråmo et al. ${ }^{17}$ For Fld2 and FNR2, the diffraction data were integrated with iMosflm ${ }^{18}$ (Fld2) or XDS ${ }^{19}$ (FNR2) and scaled and merged with Aimless. ${ }^{20}$ The protein structures were solved with molecular replacement (MR) using the Phaser $^{21}$ program with 3lzw (FNR2) and 6 fsg (Fld2) as search models. For all proteins, several cycles of refinement were carried out using first Refmac $5^{22}$ and subsequently Phenix.Refine ${ }^{23}$ in the Phenix suite. ${ }^{24}$ Model validation was performed using MolProbity. ${ }^{25}$ Visualization and model building of the protein molecule were done in Coot. ${ }^{26}$ Illustrations of the protein were prepared with PyMOL. ${ }^{27}$ The absorbed X-ray doses were calculated with the program RADDOSE-3D. ${ }^{28}$

Activity Measurements. The activity measurements and the data analysis were performed essentially as described by Lofstad et al. ${ }^{11}$ In brief, the reactions were carried out with $200 \mu \mathrm{M}$ NADPH, $0.5 \mu \mathrm{M}$ FNR (for FNR1 and FNR3) or $20 \mathrm{nM}$ FNR (for FNR2), and various concentrations of Fld. NADPH and FNR were incubated and stirred for $15 \mathrm{~min}$; Fld was added to start the reaction, or, to avoid dilution effects, the reaction was initiated by the addition of FNR. The reduction of the Flds was monitored by the disappearance of the $\mathrm{Fld}_{\mathrm{ox}}$ state at $\lambda_{\max }=$ $461 \mathrm{~nm}$ (Fld1) or $459 \mathrm{~nm}$ (Fld2) and the appearance of the Fld $_{\mathrm{sq}}$ state at $\lambda_{\max }=594 \mathrm{~nm}($ Fld1) or $592 \mathrm{~nm}($ Fld2).

Redox Potential Measurements. The redox potentials were mostly measured using the xanthine/xanthine oxidase method described by Massey. ${ }^{29}$ Buffer (50 mM HEPES pH 7.1, $100 \mathrm{mM} \mathrm{KCl}$ ), reagent stock solutions and proteins were made anaerobic on a Schlenk line before being transferred to a glovebox (Plas Laboratories 855-AC) that contained an Agilent $8453 \mathrm{UV}$-visible spectrophotometer, all under strict anaerobic conditions $\left(91 \% \mathrm{~N}_{2}, 9 \% \mathrm{H}_{2}\right.$, Praxair). In a cuvette with stirring, approximately equimolar amounts of protein and a redoxactive dye $(\approx 20 \mu \mathrm{M})$ were mixed with xanthine $(250 \mu \mathrm{M})$ and methyl viologen $(2 \mu \mathrm{M})$ in a total volume of $1 \mathrm{~mL}$. Xanthine oxidase $(9 \mu \mathrm{g} / \mathrm{mL})$ was added to initiate the reaction. The concentration of xanthine oxidase in the assay was adjusted so that the reduction was slow enough to ensure equilibrium. In addition, reference spectra of the redox dyes in their oxidized and reduced form, and of the different flavoproteins in their oxidized (ox), semiquinone (sq), and hydroquinone (hq) forms, all with known concentration, were also recorded.
The reduction of the flavoprotein and the redox dye were continuously monitored. For each recorded spectrum, the contribution/fraction $(\mathrm{a}, \mathrm{b}, \mathrm{c}, \ldots)$ of the appropriate individual reference spectra $(A, B, C, \ldots)$ to the total, composite spectrum was determined in Origin, using a nonlinear fit of this spectrum to the function $\mathrm{a} \cdot \mathrm{A}+\mathrm{b} \cdot \mathrm{B}+\mathrm{c} \cdot \mathrm{C}+$ etc. This analysis gave a series of measurements of the $\left[\right.$ dye $\left._{\text {ox }}\right],\left[\right.$ dye $\left._{\text {red }}\right],\left[\right.$ flavin $\left._{\text {ox }}\right]$, and $\left[\right.$ flavin $\left._{\mathrm{sq}}\right]$ (or $\left[\right.$ flavin $\left._{\mathrm{hq}}\right]$ ) at different time points. Plotting $\lg \frac{\left[\text { flavin }_{\mathrm{ox}}\right]}{\left[\mathrm{flavin}_{\mathrm{sq}}\right]}$ versus $\lg \frac{\left[\mathrm{dye}_{\mathrm{ox}}\right]}{\left[\mathrm{dye}_{\mathrm{red}}\right]}$ gives a linear plot where the intercept of the ordinate has $\left[\right.$ dye $\left._{\mathrm{ox}}\right]=\left[\right.$ dye $\left._{\mathrm{red}}\right]$, resulting in the Nernst equation being $E_{\mathrm{ox} / \mathrm{sq}}=E_{\text {dye }}-\frac{0.06015}{n} \lg \frac{\left[\mathrm{flavin}_{\mathrm{ox}}\right]}{\left[\mathrm{flavin}_{\mathrm{sq}}\right]}$ at $30{ }^{\circ} \mathrm{C}, n=1$ for $E_{\mathrm{ox} / \mathrm{sq}}$ and $E_{\mathrm{sq} / \mathrm{hq}}, n=2$ for $E_{\mathrm{ox} / \mathrm{hq}}$. An example of the analysis is shown in Figure $\mathrm{S} 7$.

For most of the proteins, the dye Safranine $\mathrm{O}$ was used to obtain a first estimate of the protein's midpoint potential. If the calculated midpoint potential of the flavoprotein was not within $30-40 \mathrm{mV}$ of the redox potential of Safranin O, other dyes were tested until the redox potentials of the dye and the protein were within $30-40 \mathrm{mV}$. The following dyes were ultimately used: ${ }^{29,30}$ Safranine O $E_{\mathrm{m}}=-289 \mathrm{mV}$ (Sigma-Aldrich) for $\mathrm{NrdI}$ ox/sq and sq/hq; 2-hydroxy-1,4-naphthoquinone $E_{\mathrm{m}}=-145 \mathrm{mV}$ (Sigma) for Fld1 and Fld2 ox/sq; anthraquinone2-sulfonate $E_{\mathrm{m}}=-225 \mathrm{mV}$ (Aldrich) for FNR1 ox/sq; phenosafranine $E_{\mathrm{m}}=-252 \mathrm{mV}$ (Sigma-Aldrich) for FNR3 ox/hq.

Redox titrations with dithionite and benzyl viologen, $E_{\mathrm{m}}=$ $-359 \mathrm{mV}$ (Sigma), were carried out to determine the Fld 1 and Fld2 sq/hq and FNR2 ox/sq redox potentials. The equilibrium concentrations of each redox component at different time points during the titration were determined, similarly to the procedure described above.

Calculation of Conserved Residues, Phylogenetic Analysis, and Structural Sequence Alignment. To evaluate the degree of conservation of residues in the FNRs and Flds, ConSurf $^{31-34}$ was run on each FNR1, FNR2, Fld1, Fld2, and $\mathrm{NrdI}$ structure. The runs were based on the homologue search algorithm HMMER, searching sequences from UniRef90, and multiple sequence alignment with MAFFT. ConSurf uses the 150 most homologous sequences for each query sequence to map the conservation on a 9-bin scale from turquoise (most variable) to maroon (most conserved). The conservation color coding for each protein was then mapped onto the 5 crystal structures.

Phylogenetic analyses on FNR1 and on FNR2 were performed in Jalview ${ }^{35}$ on the 150 sequences selected in the ConSurf runs. Clustal $\mathrm{O}^{36}$ was used for sequence alignment, and average distances in the phylogenetic tree were calculated with Blossum 62. Some sequences that were shorter than FNR1 and FNR2 were not included in the phylogenetic analysis, as they did not contain the C-terminal residues found in both FNR1 and FNR2.

Table 1. Kinetic Parameters of Flavodoxin Reduction (Fld1-2 and NrdI) by Different Flavodoxin Reductases (FNR1-3) from B. cereus

\begin{tabular}{|c|c|c|c|c|c|c|c|c|c|}
\hline & \multicolumn{3}{|c|}{ FNR1 } & \multicolumn{3}{|c|}{ FNR2 } & \multicolumn{3}{|c|}{ FNR3 } \\
\hline & $\begin{array}{c}k_{\text {cat }} \\
\left(\min ^{-1}\right)\end{array}$ & $\begin{array}{c}K_{\mathrm{M}} \\
(\mu \mathrm{M})\end{array}$ & $\begin{array}{c}k_{\mathrm{caf}} / K_{\mathrm{M}} \\
\left(\mu \mathrm{M}^{-1} \min ^{-1}\right)\end{array}$ & $\begin{array}{c}k_{\text {cat }} \\
\left(\min ^{-1}\right)\end{array}$ & $\begin{array}{c}K_{\mathrm{M}} \\
(\mu \mathrm{M})\end{array}$ & $\begin{array}{c}k_{\mathrm{cat}} / K_{\mathrm{M}} \\
\left(\mu \mathrm{M}^{-1} \min ^{-1}\right)\end{array}$ & $\begin{array}{c}k_{\text {cat }} \\
\left(\min ^{-1}\right)\end{array}$ & $\begin{array}{c}K_{\mathrm{M}} \\
(\mu \mathrm{M})\end{array}$ & $\begin{array}{c}k_{\mathrm{cat}} / K_{\mathrm{M}} \\
\left(\mu \mathrm{M}^{-1} \min ^{-1}\right)\end{array}$ \\
\hline $\operatorname{NrdI}^{a}$ & $8.0 \pm 0.1$ & $2.7 \pm 0.2$ & $3.0 \pm 0.2$ & $100 \pm 4$ & $61 \pm 5$ & $1.6 \pm 0.2$ & $2.94 \pm 0.04$ & $0.74 \pm 0.06$ & $3.9 \pm 0.4$ \\
\hline Fld 1 & $7.3 \pm 0.5$ & $4.7 \pm 0.8$ & $1.5 \pm 0.3$ & $2778 \pm 401$ & $25 \pm 7$ & $111 \pm 47$ & $2.4 \pm 0.2$ & $4.4 \pm 1.3$ & $0.6 \pm 0.2$ \\
\hline Fld 2 & $42 \pm 8$ & $60 \pm 22$ & $0.7 \pm 0.4$ & $9125 \pm 1450$ & $13 \pm 5$ & $701 \pm 360$ & $2.9 \pm 0.3$ & $8 \pm 2$ & $0.4 \pm 0.2$ \\
\hline
\end{tabular}

${ }^{a}$ Reduction rates are from ref 11 . 
Table 2. Reduction Potentials of the B. cereus FNR1-3, Fld1-2, and NrdI

\begin{tabular}{lccc} 
protein & $E_{\text {ox } / \mathrm{sq}}(\mathrm{mV})$ & $E_{\mathrm{sq} / \mathrm{hq}}(\mathrm{mV})$ & $E_{\mathrm{ox} / \mathrm{hq}}(\mathrm{mV})$ \\
NrdI & -252 & -309 & \\
Fld1 & -149 & -380 & \\
Fld2 & -127 & -385 & \\
FNR1 & -228 & $\mathrm{n} / \mathrm{a}$ & \\
FNR2 & -332 & $\mathrm{n} / \mathrm{a}$ & \\
FNR3 & $\mathrm{n} / \mathrm{a}$ & $\mathrm{n} / \mathrm{a}$ & -242 \\
\hline
\end{tabular}

For structural sequence alignment of the $5 \mathrm{Fld} / \mathrm{NrdI}$ and FNR structures, DALI searches were performed. ${ }^{37}$ The secondary structure assignments (helix, strand, coil) were performed with DSSP by the DALI protein structure comparison server.

Calculation of Electrostatic Surface Potential. The electrostatic surface potentials were calculated using the
APBS-plugin module ${ }^{38}$ in PyMOL. The charges were assigned using PDB 2PQR before the APBS module was run to calculate the electrostatic properties.

To make the model of FNR1 in the open conformation of FNR2, the FNR1 sequence was homology modeled with SWISS-MODEL ${ }^{39-43}$ using the FNR2 structure (PDB ID: 6gas) as a template. The FAD-binding domain of the original FNR1 structure (PDB ID: 6gar) was aligned to the modeled FNR1 structure, and the FAD groups and the $\mathrm{C}$-terminal helices from the original structure were added to the homology modeled FNR1. The surface potential was calculated as described above.

\section{RESULTS AND DISCUSSION}

FNR2 is the Most Efficient Fld Reductase with the FNR2-FId2 Pair Being the Most Efficient Electron Transfer Pair. The rate of reduction of Fld1 and Fld2 by

Table 3. Crystal Data Collection and Refinement Statistics for FNR1, FNR2, and Fld2

\begin{tabular}{|c|c|c|c|}
\hline & $\mathrm{FNR}^{a}{ }^{a}$ & FNR2 & Fld2 \\
\hline \multicolumn{4}{|c|}{ Data Collection } \\
\hline $\mathrm{X}$-ray source & ID29, ESRF & ID30A-3, ESRF & X10SA, SLS \\
\hline wavelength $(\AA)$ & 0.9763 & 0.9677 & 0.9999 \\
\hline space group & $P 2_{1} 22_{1}$ & $P 3_{2} 2$ & $\mathrm{C} 222_{1}$ \\
\hline$a, b, c(\AA)$ & $57.2,95.0,164.3$ & $109.7,109.7,242.5$ & $101.5,125.4,59.4$ \\
\hline$\alpha, \beta, \gamma(\operatorname{deg})$ & $90,90,90$ & $90,90,120$ & $90,90,90$ \\
\hline total rotation range $(\mathrm{deg})$ & 119.1 & 235 & 130 \\
\hline exposure time per image $(\mathrm{s})$ & 0.037 & 0.01 & 0.1 \\
\hline flux $(\mathrm{ph} / \mathrm{s})$ & $6.4 \times 10^{11}$ & $5.8 \times 10^{11}$ & $9.44 \times 10^{11}$ \\
\hline beam size $\left(\mu \mathrm{m}^{2}\right)$ & $30 \times 50$ & $15 \times 15$ & $120 \times 120$ \\
\hline crystal size $\left(\mu \mathrm{m}^{3}\right)$ & $50 \times 50 \times 150$ & $90 \times 90 \times 90$ & $30 \times 30 \times 70$ \\
\hline av absorbed X-ray dose (MGy) (exposed regions) & 2.2 & 7.6 & 3.2 \\
\hline av diffraction weighted absorbed X-ray dose (MGy) & 2.1 & 7.1 & 1.6 \\
\hline resolution range $(\AA)$ & $54.8-2.5(2.6-2.5)$ & $48.50-2.40(2.46-2.40)$ & $62.7-2.5(2.6-2.5)$ \\
\hline total no. of reflections & 137105 & 877673 & 55394 \\
\hline no. of unique reflections & 31641 & 66924 & 13361 \\
\hline$R_{\text {meas }}$ & $0.140(0.597)$ & $0.145(1.076)$ & $0.200(1.113)$ \\
\hline$R_{\text {merge }}$ & $0.130(0.632)$ & $0.139(1.028)$ & $0.175(1.123)$ \\
\hline completeness (\%) & $99.5(98.7)$ & $100(100)$ & $99.8(99.9)$ \\
\hline multiplicity & $4.3(4.1)$ & $13.1(11.3)$ & $4.1(4.3)$ \\
\hline$[I / \sigma(I)]$ & $8.8(2.6)$ & $11.4(2.0)$ & $6.4(1.6)$ \\
\hline$C C_{1 / 2}$ & $0.99(0.79)$ & $0.998(0.749)$ & $0.992(0.633)$ \\
\hline \multicolumn{4}{|c|}{ Refinement Statistics } \\
\hline$R_{\text {work }} / R_{\text {free }}$ & $18.6 / 23.5$ & $20.7 / 26.2$ & $23.5 / 29.3$ \\
\hline mean protein/solvent isotropic $B$ factor $\left(\AA^{2}\right)$ & $42.7 / 32.9$ & $56.7 / 48.8$ & $44.5 / 31.7$ \\
\hline protein assembly in asymmetric unit (AU) & 1 dimer & 2 dimers & 2 monomers \\
\hline protein residues (by chain) & $\begin{array}{l}689 \text { (A: } 4-349, \text { B: } \\
\text { 5-347) }\end{array}$ & $\begin{array}{l}1306(\mathrm{~A}: 6-331, \mathrm{~B}: 5-331, \mathrm{C}: 5-331, \mathrm{D}: \\
6-331)\end{array}$ & $\begin{array}{l}292 \text { (A: } 9-154, \text { B: } \\
\text { 9-154) }\end{array}$ \\
\hline ligand atoms & 132 & 216 & 80 \\
\hline added water atoms & 88 & 44 & 10 \\
\hline solvent content $(\%)$ & 57.3 & 57.6 & 55.6 \\
\hline Matthews coefficient $\left(\AA^{3} / \mathrm{Da}\right)$ & 2.9 & 2.9 & 2.8 \\
\hline Ramachandran plot: most favored/allowed & $94.9 / 4.4$ & $95.7 / 3.8$ & $97.9 / 1.7$ \\
\hline RMSD bond lengths $(\AA)$ & 0.008 & 0.011 & 0.004 \\
\hline RMSD bond angles (deg) & 1.28 & 1.26 & 0.95 \\
\hline $\begin{array}{l}\text { estimated overall coordinate error based on maximum } \\
\text { likelihood }(\AA)\end{array}$ & 0.28 & 0.38 & 0.36 \\
\hline PDB ID & 6gar & 6gas & 6gaq \\
\hline
\end{tabular}

${ }^{a}$ The data collection statistics for FNR1 are from the work of Skråmo et al. ${ }^{17}$ 


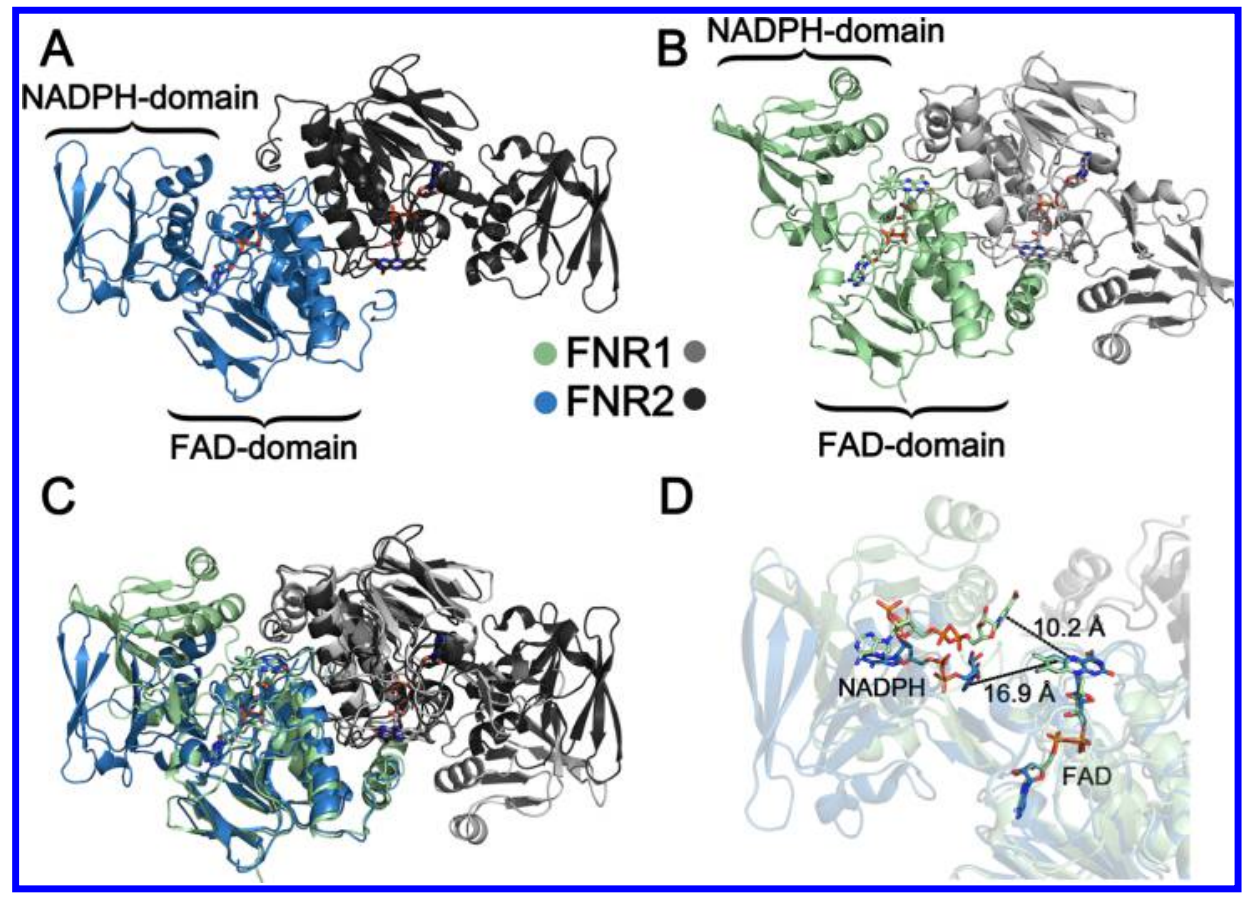

Figure 1. (A, B) Crystal structure of FNR2 (A) and FNR1 (B). The protomers are colored blue and black (FNR2) or green and gray (FNR1), and the NADPH- and FAD-binding domains are indicated with brackets. (C) Overlay of the crystal structures of FNR1 and FNR2. (D) The tentative NADPH-binding site and distance to the FAD cofactor in FNR1 and FNR2. The NADPH molecules are docked into the structures based on the NADPH-binding site in the B. subtilis YumC structure (PDB ID: 3lzw). The cofactors are shown in stick representation.

the three different FNRs were measured and compared with the published reduction rates of $\mathrm{NrdI}$ by the same reductases ${ }^{11}$ (Table 1). For all three flavodoxins, the highest turnover rates are achieved with FNR2; in particular, FNR2 reduces Fld2 and Fld 1 at a 91 -fold and 28 -fold higher rate, respectively, than the third fastest FNR-Fld pair, FNR2 and NrdI. In contrast to the other FNR-Fld pairs, where there is no reduction of the Fld under aerobic conditions, the reaction between FNR2 and Fld2 even proceeds at a high rate under aerobic conditions, with a $k_{\text {cat }}$ of $11943 \pm 1132 \mathrm{~min}^{-1}$. Compared to steady-state kinetic data from other FNR-Fld systems, the FNR2-Fld2 pair has a comparable reduction rate to the E. coli FNR-Fld system, ${ }^{10}$ which has a reported $V_{\max }$ of $272 \pm 11.5 \mathrm{~min}^{-1}$ at an FNR concentration of $16.65 \mathrm{nM}$, giving a $k_{\text {cat }}$ of $17000 \mathrm{~min}^{-1}$. Thus, even if the B. cereus FNRs may transfer electrons to all of the Flds (Scheme 1), FNR2 is clearly the most efficient Fld reductase. This indicates that the FNR-Fld interaction in $B$. cereus is selective.

The Protein Reduction Potentials Underpin the Reduction Kinetics. None of the FNR-Fld complexes elute as a single peak on a size exclusion column, not even after overnight incubation of the complex before gel filtration (results not shown). Weak binding is typical of electron transfer complexes, ${ }^{44}$ and our results are also in line with the weak dissociation constants we have observed previously for the FNRNrdI binding. ${ }^{11}$ The proteins must therefore have other, differing properties that can help explain the reduction kinetics. One obvious parameter to investigate is the driving force of the electron transfer reaction, i.e., the reduction potential of the proteins. Indeed, the measured redox potentials, shown in Table 2, substantiate the reduction kinetics studies. Of the FNRs, FNR2 is the best electron donor, with an ox/sq reduction potential of $-332 \mathrm{mV}$. Conversely, Fld2 is the best electron acceptor, with an ox/sq reduction potential of $-127 \mathrm{mV}$, the least negative of all the measured potentials. Yet, it is unlikely that the difference in reduction rates is fully explained by the variation in the proteins' reduction potentials alone. Additional features must also contribute to the reaction rates. One such possible, defining feature is the rigidity of the hinge region between the FNR $\mathrm{NADPH}$ - and FAD-binding domains, which will be further discussed below.

The Flavodoxins Are Structurally Similar. The crystal structure of Flavodoxin 2, presented here, supplements the already published crystal structures of $B$. cereus Fld $1^{14}$ and $\mathrm{NrdI}^{15}$ (Table 3). Comparing the crystal structure of Fld2 with the Fld 1 structure, the flavodoxins are structurally very similar. They have comparable active sites and 3D structures, a fivestranded $\beta$-sheet surrounded by $\alpha$-helices (Figure S1). Given this structural similarity, it is not surprising that the Flds have very similar redox potentials, and that both Fld 1 and Fld 2 are good redox partners for FNR2. NrdI, however, is not classified as a flavodoxin, but as a flavodoxin-like protein, and has a lower sequence similarity with the Flds (Figure S2). We therefore see more differences in the active site compared to the flavodoxins, but these differences are conserved within NrdIs from different organisms (Figure S1).

The FNRs Have Different Conformations, Which Likely Affects the Catalytic Mechanism. We have also solved the crystal structures of FNR1 and FNR2 (Table 3), shown in Figure 1A,B. Just like bacterial TrxRs, the TrxR-like FNRs are homodimers, and each monomer has a FAD- and a $\mathrm{NADPH}$-binding domain. The NADPH-binding domain is connected to the FAD-binding domain by a short hinge that consists of an antiparallel $\beta$-sheet, and the domains can rotate relative to each other due to the lack of interactions between the two domains. ${ }^{45}$ From Figure 1C, it is evident that the FNRs have crystallized in different conformations. Superimposing the FAD-binding domains of the two proteins, we find that the $\mathrm{NADPH}$-binding domain of FNR1 is rotated $60^{\circ}$ relative to the NADPH-binding domain in FNR2 (Table S1). ${ }^{46}$ 


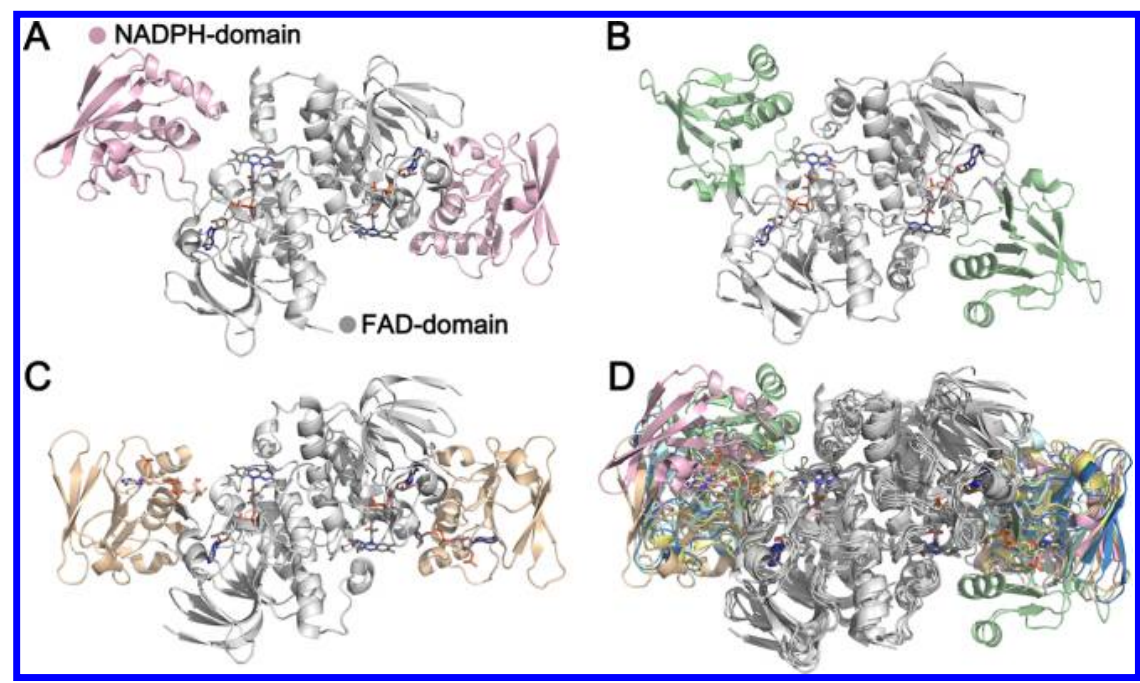

Figure 2. (A-C) Crystal structures of C. tepidum FNR, 3ab1 (A), B. cereus FNR1 (B), B. subtilis YumC, 3lzx (C), showing the different rotation in the FNR NADPH-binding domains (colored) relative to the FAD-binding domain (gray). (D) Overlay of the structures of TrxR-like FNR from the Protein Data Bank. The relative rotation angles are provided in Table S1. Protein name, PDB IDs, and corresponding colors: B. cereus FNR1, green; B. cereus FNR2, blue; B. subtilis YumC, 3lzx, wheat; C. tepidum FNR 3ab1, pink; T. thermophilus TrxR-like protein, 2zbw, cyan; B. subtilis YumC, 3lzw, yellow. The FAD and NADPH cofactors are shown in stick representation.

In the structurally homologous TrxRs, the NADPH-binding domain and the FAD-binding domain are also found in two distinct conformations, depending on the oxidation state of the $\mathrm{FAD}$. In one conformation, the FAD cofactor is just opposite NADPH and is thus in an ideal position for hydride transfer by $\mathrm{NADPH}$, whereas the active site disulfide is exposed to the solvent and therefore able to reduce the substrate, thioredoxin. Once $\mathrm{FAD}$ is reduced, the NADPH-binding domain rotates and the, now oxidized, TrxR active site disulfide can access the reduced $\mathrm{FAD}$, with subsequent reduction of the disulfide and rearrangement of the two domains. ${ }^{47-49}$ Mutational studies have shown that a flexible hinge region is important for the catalytic function of the TrxR-like FNRs, too. A study on a TrxRlike FNR from Chlorobaculum tepidum found that mutating two conserved residues in the hinge region, Gly260 and Gly266, to proline significantly decreased the reactivity of the FNR ${ }^{45}$ Interestingly, the FNRs do not seem to be restricted to only two conformations, as with the TrxRs. An overlay of all the TrxR-like FNRs in the Protein Data Bank (Figure 2) shows that the FNRs have crystallized in various conformations, although the proteins seem to favor either a closed conformation similar to FNR1, or a more open conformation, as FNR2. The relative rotation angles are provided in Table S1.

Despite this conformational flexibility, none of the FNRs have crystallized in a conformation that would allow for hydride transfer from NADPH to FAD. Whereas electron tunnelling can take place over distances up to $14 \AA$, hydride transfer between nicotinamides and flavins is expected to occur at distances of $1-2 \AA^{50}$ In this work, FNR2 was cocrystallized with $\mathrm{NADP}^{+}$; yet there is no electron density for $\mathrm{NADP}^{+}$in the FNR2 structure. Thus, the structure of the Bacillus subtilis FNR YumC is the only published structure of a TrxR-like FNR where $\mathrm{NADP}^{+}$is bound. ${ }^{51}$ There, the NADPH molecule is over $15 \AA$ away from the FAD molecule, which is too far away for efficient hydride transfer. This suggests that, similarly to TrxR, a large-scale conformational rearrangement must take place for FAD to be reduced by NADPH in the TrxR-like FNRs. ${ }^{50,51}$ We have docked NADPH into the FNR1 and FNR2 structures, based on its binding site in the B. subtilis YumC

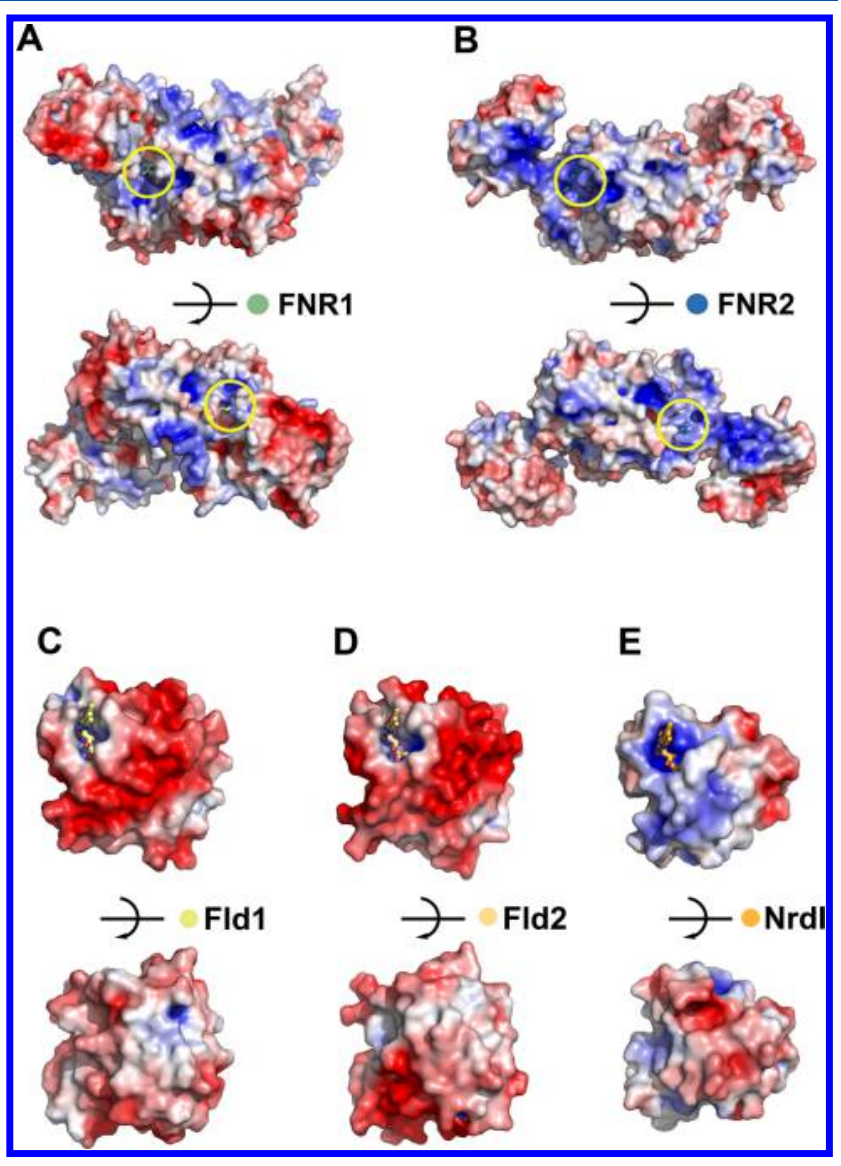

Figure 3. $\pm 5 \mathrm{kT} / \mathrm{e}$ electrostatic potential of the B. cereus FNRs (A, B) and Flds (C-E) plotted on the solvent-accessible surface. The position of the FAD cofactors in FNR1 and FNR2 is marked with a yellow circle.

structure (Figure 1D). In FNR1, the FNR with a closed conformation, NADPH is $10.2 \AA$ away from FAD (flavin N5 to NADPH C4), whereas in FNR2, the more open FNR, $\mathrm{NADPH}$ is $16.9 \AA$ away (flavin N5 to NADPH C4). Thus, it seems like it is the closed conformation of FNR1 that 


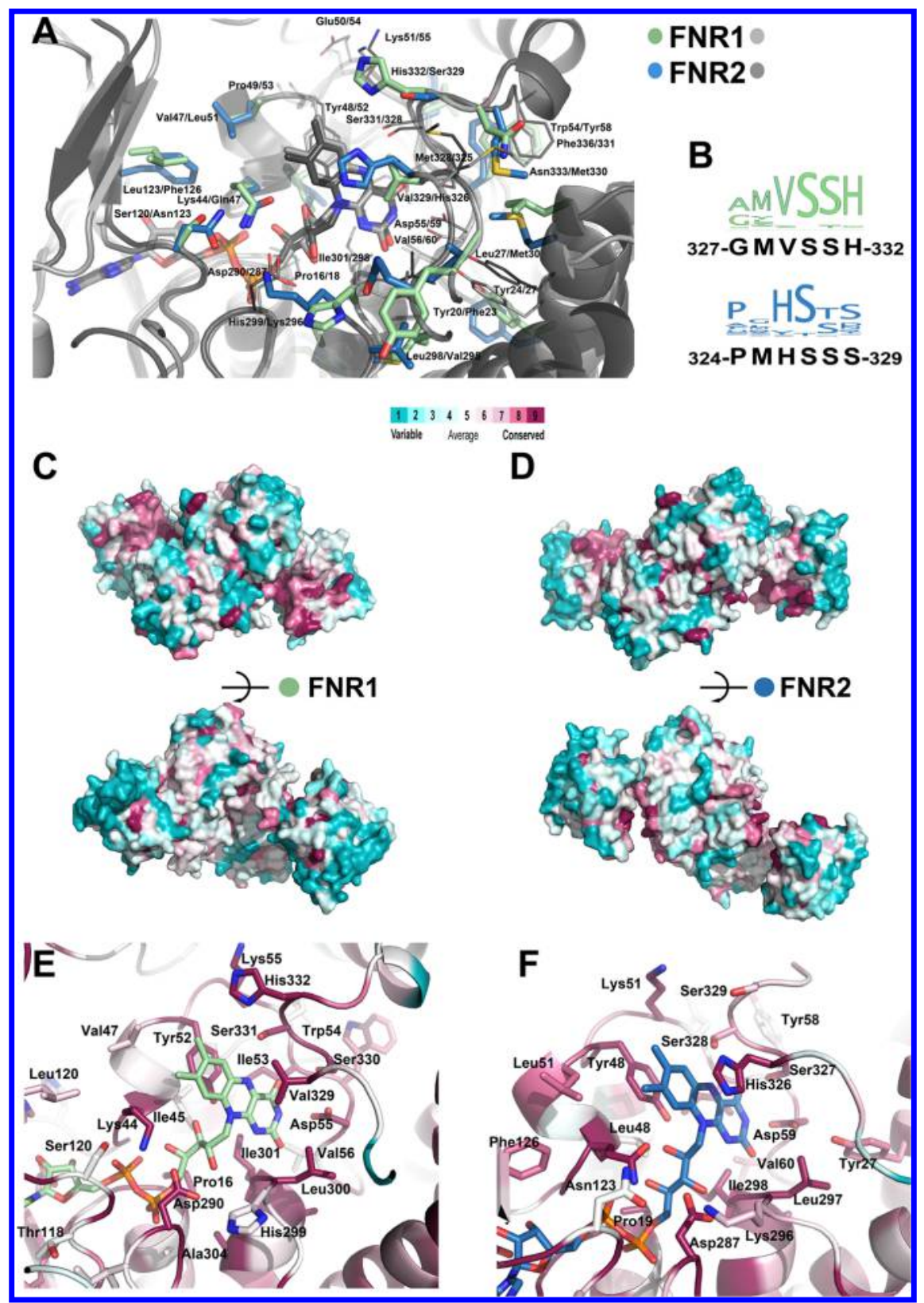

Figure 4. (A) Comparison of the active sites of FNR1 and FNR2. Identical residues in the overlaid structures are shown with lines and are colored in shades of gray, while different residues are shown with sticks and are colored in green and blue. (B) Extracts of the consensus sequence from the multiple sequence alignment of the FNR1 (green) and FNR2 (blue) phylogenetic clades (shown in Figures S5 and S6). The FNR1 and FNR2 sequences are shown in black. (C-F) The degree of conservation of the FNR surface $(C, D)$ and active site (E, F) residues has been evaluated using ConSurf. Variable residues are colored in turquoise, and highly conserved residues are colored in maroon.

approaches the conformation that the FNR must be in to allow for hydride transfer.

In the closed conformation displayed by FNR1, it is, however, more difficult to imagine substrate binding without a conformational change. In contrast, in the open conformation of FNR2, it is easy to see how a flavodoxin can fit for productive electron transfer (assuming binding with the FAD and FMN cofactors juxtaposed $\left.{ }^{8}\right)$. In the absence of a protein-protein complex crystal structure, we have docked the different Flds to the FNRs (Figure S3). Of the resulting structures, it is only in the FNR2-Fld2 complex that the Fld docks close to the FADbinding site, supporting our hypothesis about the conformational changes that likely take place during the FNR catalytic cycle. This conformational flexibility is thus a unique feature of the
TrxR-like FNRs, in contrast to the monomeric FNRs, which are characterized by a rigid structure, where $\mathrm{NADPH}$ is positioned close to the FAD so that there are no large structural movements upon binding of Flds or ferredoxins (Fds). The conformational changes could also give yet another explanation for the differing reduction rates. The amino acid sequence in the hinge region varies considerably between FNR1 and FNR2 (Figure S2). This disparity could influence the free energy of rotation of the two proteins, and, consequently, the electron transfer rate.

Electron transfer proteins are typically guided toward each other by electrostatic interactions. ${ }^{44,52}$ Figure 3 shows the calculated electrostatic surface potentials of the FNRs and Flds. The Flds have acidic residues surrounding the FMN cofactor, whereas NrdI has basic residues in this region. The FNRs have 
differing surface charges, too. FNR1 has small basic patches in several places on the surface, whereas FNR2 has a single, but larger, basic patch around its FAD cofactor. We have also homology modeled FNR1 in the conformation of FNR2 (Figure S4) to see how a conformational change affects the solvent-accessible surface. In the modeled open conformation, more of the basic residues around the FNR1 FAD cofactor are accessible, but the basic patch is still smaller than the patch on FNR2. The single basic patch on FNR2 probably increases the chances of forming a productive electron transfer complex, which could explain, in part, the faster turnovers achieved by FNR2 compared to FNR1.

FNR1 Belongs to a Novel Class of TrxR-like FNRs. An intriguing difference between FNR1 and FNR2 became apparent upon inspection of the conserved residues surrounding the FAD cofactor in the FNR active site. In all of the published structures of TrxR-like FNRs to date, three residues in the C-terminal subdomain, a residue with aromatic character (His, Tyr, or Phe) followed by two aliphatic hydroxyl-containing residues (Ser or Thr), have been found to stabilize the FAD cofactor by $\pi-\pi$ interactions and hydrogen-bond interactions. ${ }^{45,51}$ In fact, these residues have been suggested to be a common structural feature of TrxR-like FNRs. ${ }^{45}$ However, in FNR1, the aromatic residue is replaced by a Val (Figure 4A). Furthermore, we find that this residue is actually conserved in several FNRs (Figure 4B and Figure S5). We have used ConSurf ${ }^{31-34}$ to map the conserved residues onto the FNR structures. Figure $4 \mathrm{C}-\mathrm{F}$ shows that the FNRs have conserved active sites, but that the other residues are variable. The homologous FNR sequences are grouped into two phylogenetic clades (Figure S6); one class where His, Tyr, or Phe is stacked opposite the $\mathrm{FAD}$ on the re face, and one where $\mathrm{Val}$ is positioned on the FAD re face (Figure 4A,E,F). FNR2 belongs to the first group and FNR1 belongs to the latter (Figures S5 and S6). The FNRs in the group containing FNR1 also have a slightly longer amino acid sequence than those in the FNR2 group, resulting in a longer C-terminal helix (Figure S5).

The interchange of the FAD-stacking residues might explain the large differences in the efficiency of the Fld reduction by FNR1 and FNR2. Also, it is possible that the class that FNR1 belongs to has another endogenous substrate than Fld, for example, ferredoxin. Studies where the stacking Phe337 (C. tepidum) or His324 (B. subtilis) residue has been mutated to other residues (Ser/His/Tyr or Ser/Phe, respectively) do not show large changes in reactivity or nucleotide specificity of the enzyme, ${ }^{4,51}$ and it has consequently been suggested that the residue shields $\mathrm{FAD}$ from exposure to solvent during catalysis. ${ }^{45}$ The role of the FAD-stacking residue, be it a residue with aromatic character or $\mathrm{Val}$, must therefore be investigated in future studies.

TrxR-like FNRs are found in several bacteria and archaea; however, our phylogenetic analysis (Figures S5 and S6) shows that the FNRs that are grouped with FNR1 are solely from the Firmicutes phylum, whereas the FNRs that cluster with FNR2 are from Firmicutes, Proteobacteria, Actinobacteria, and green sulfur bacteria. Therefore, the FNR1-type FNRs seem to be specific for Firmicutes, whereas the FNR2-type FNRs might be more general. The presence of the different FNRs, Flds, and Fds among the Firmicutes species also varies. For example, B. cereus contains genes encoding for FNR1 and FNR2, two Flds, and two Fds, and Lactococcus lactis only contains an FNR2-type FNR, a Fld, and a small Fd, while Staphylococcus aureus has an FNR1-type FNR and a Fd, but no Flds. This might point toward the FNR1 group of FNRs favoring reduction of Fds, but further biochemical and physiological studies must be carried out to elucidate the biological significance of these species-related differences.

Concluding Remarks. Enzyme kinetics and bioinformatics show that the TrxR-like FNRs should be divided into two subclasses, based on the identity of the isoalloxazine-ringstacking residue. Furthermore, from the data presented here it is evident that there are large differences in the rate of flavodoxin reduction by the different FNRs in B. cereus and that a large-scale domain rearrangement must take place during the catalytic cycle of the TrxR-like FNRs, analogous to the TrxRs and in contrast to the monomeric FNRs. FNR2 is the most efficient reductase for both of the flavodoxins, and the FNR2Fld2 pair is particularly efficient. This knowledge is useful in itself with regards to biotechnological applications of the FNRFld system; however, it also raises a physiological question about what the different roles of the B. cereus FNRs and Flds are in vivo. Of the $B$. cereus FNRs, FNR2 has the highest sequence identity to the FNR YumC from B. subtilis. ${ }^{11}$ YumC has been found to be an essential protein in B. subtilis, ${ }^{53}$ and given that the flavodoxin reduction rates achieved by FNR2 is much higher than those of the other FNRs, it could be that FNR2 functions as the principal flavodoxin reductase in B. cereus. This possibility, as well as the function of the other FNRs and Flds, needs to be explored in future studies.

\section{ASSOCIATED CONTENT}

\section{Supporting Information}

The Supporting Information is available free of charge on the ACS Publications website at DOI: 10.1021/acs.biochem.8b00674.

Experimental details; comparison of the Fld crystal structures; structural alignments; relative FNR rotation angles; FNR-Fld docking models; FNR1 homology model; multiple sequence alignment and phylogenetic tree (PDF)

\section{AUTHOR INFORMATION}

\section{Corresponding Authors}

*E-mail: ingvild.gudim@ibv.uio.no. Tel: +47-22854631.

*E-mail: h.p.hersleth@ibv.uio.no.

ORCID $\odot$

Hans-Petter Hersleth: 0000-0002-1142-9983

\section{Funding}

This work has been supported by grants from The Research Council of Norway (231669) and from The University of Oslo.

\section{Notes}

The authors declare no competing financial interest.

\section{ACKNOWLEDGMENTS}

Dr. B. Dalhus provided access to crystallization screening at the Regional Core Facility for Structural Biology and Bioinformatics at the South-Eastern Norway Regional Health Authority (2015095). We gratefully acknowledge the SLS and the ESRF for providing beam time and for excellent technical assistance. $\mathrm{X}$-ray coordinates and structure factors have been deposited in the PDB Database (PDB IDs: 6gar (FNR1), 6gas (FNR2), 6gaq (Fld2)). 


\section{ABBREVIATIONS}

Fd, ferredoxin; Fld, flavodoxin; FNR, ferredoxin/flavodoxin $\mathrm{NADP}^{+}$reductase; GR, glutathione reductase; hq, hydroquinone; ox, oxidized; sq, semiquinone; TrxR, thioredoxin reductase.

\section{REFERENCES}

(1) Sancho, J. (2006) Flavodoxins: sequence, folding, binding, function and beyond. Cell. Mol. Life Sci. 63, 855-864.

(2) Nordlund, P., and Reichard, P. (2006) Ribonucleotide Reductases. Annu. Rev. Biochem. 75, 681-706.

(3) Wang, Z.-Q., Lawson, R. J., Buddha, M. R., Wei, C.-C., Crane, B. R., Munro, A. W., and Stuehr, D. J. (2007) Bacterial Flavodoxins Support Nitric Oxide Production by Bacillus subtilis Nitric-oxide Synthase. I. Biol. Chem. 282, 2196-2202.

(4) Birch, O. M., Fuhrmann, M., and Shaw, N. M. (1995) Biotin Synthase from Escherichia coli, an Investigation of the Low Molecular Weight and Protein Components Required for Activity in Vitro. L Biol. Chem. 270, 19158-19165.

(5) Ifuku, O., Koga, N., Haze, S.-I., Kishimoto, J., and Wachi, Y. (1994) Flavodoxin is Required for Conversion of Dethiobiotin to Biotin in Escherichia coli. Eur. I. Biochem. 224, 173-178.

(6) Blaschkowski, H. P., Knappe, J., Ludwig-Festl, M., and Neuer, G. (1982) Routes of Flavodoxin and Ferredoxin Reduction in Escherichia coli. Eur. I. Biochem. 123, 563-569.

(7) Fujii, K., and Huennekens, F. M. (1974) Activation of Methionine Synthetase by a Reduced Triphosphopyridine Nucleotide-dependent Flavoprotein System. I. Biol. Chem. 249, 6745-6753. (8) Hall, D. A., Vander, K. C. W., Stasik, C. N., Stevens, S. Y., Zuiderweg, E. R., and Matthews, R. G. (2001) Mapping the interactions between flavodoxin and its physiological partners flavodoxin reductase and cobalamin-dependent methionine synthase. Proc. Natl. Acad. Sci. U. S. A. 98, 9521-9526.

(9) Aliverti, A., Pandini, V., Pennati, A., de Rosa, M., and Zanetti, G. (2008) Structural and functional diversity of ferredoxin-NADP ${ }^{+}$ reductases. Arch. Biochem. Biophvs. 474, 283-291.

(10) McIver, L., Leadbeater, C., Campopiano, D. J., Baxter, R. L., Daff, S. N., Chapman, S. K., and Munro, A. W. (1998) Characterization of flavodoxin NADP+ oxidoreductase and flavodoxin; key components of electron transfer in Escherichia coli. Eur. I. Biochem. $257,577-585$.

(11) Lofstad, M., Gudim, I., Hammerstad, M., Røhr, Å. K., and Hersleth, H.-P. (2016) Activation of the Class Ib Ribonucleotide Reductase by a Flavodoxin Reductase in Bacillus cereus. Biochemistry 55, 4998-5001.

(12) Lawson, R. J., von Wachenfeldt, C., Haq, I., Perkins, J., and Munro, A. W. (2004) Expression and Characterization of the Two Flavodoxin Proteins of Bacillus subtilis, YkuN and YkuP: Biophysical Properties and Interactions with Cytochrome P450 Biol. Biochemistry 43, 12390-12409.

(13) Mandai, T., Fujiwara, S., and Imaoka, S. (2009) Construction and engineering of a thermostable self-sufficient cytochrome P450. Biochem. Biophvs. Res. Commun. 384, 61-65.

(14) Gudim, I., Lofstad, M., van Beek, W., and Hersleth, H.-P. (2018) High-resolution crystal structures reveal a mixture of conformers of the Gly61-Asp62 peptide bond in an oxidized flavodoxin from Bacillus cereus. Protein Sci..

(15) Røhr, Å. K., Hersleth, H.-P., and Andersson, K. K. (2010) Tracking Flavin Conformations in Protein Crystal Structures with Raman Spectroscopy and QM/MM Calculations. Angew. Chem. Int. Ed. 49, 2324-2327.

(16) Edebo, L. (1960) A new press for the disruption of microorganisms and other cells. I. Biochem. Microbiol. Technol. Eng. 2, 453479.

(17) Skråmo, S., Hersleth, H.-P., Hammerstad, M., Andersson, K. K., and Røhr, Å. K. (2014) Cloning, expression, purification, crystallization and preliminary X-ray diffraction analysis of a ferredoxin/ flavodoxin- $\mathrm{NADP}(\mathrm{H})$ oxidoreductase (Bc0385) from Bacillus cereus. Acta Crystallogr. Sect. F: Struct. Biol. Commun. 70, 777-780.

(18) Battye, T. G. G., Kontogiannis, L., Johnson, O., Powell, H. R., and Leslie, A. G. W. (2011) iMOSFLM: a new graphical interface for diffraction-image processing with MOSFLM. Acta Crystallogr., Sect. D: Biol. Crystallogr. 67, 271-281.

(19) Kabsch, W. (2010) XDS. Acta Crystallogr., Sect. D: Biol. Crystallogr. 66, 125-132.

(20) Evans, P. R., and Murshudov, G. N. (2013) How good are my data and what is the resolution? Acta Crystallogr., Sect. D: Biol. Crystallogr. 69, 1204-1214.

(21) McCoy, A. J., Grosse-Kunstleve, R. W., Adams, P. D., Winn, M. D., Storoni, L. C., and Read, R. J. (2007) Phaser crystallographic software. L. Appl. Crystallogr. 40, 658-674.

(22) Murshudov, G. N., Skubák, P., Lebedev, A. A., Pannu, N. S., Steiner, R. A., Nicholls, R. A., Winn, M. D., Long, F., and Vagin, A. A. (2011) REFMAC5 for the refinement of macromolecular crystal structures. Acta Crustallogr. Sect. D: Biol. Crustallogr. 67, 355-367.

(23) Afonine, P. V., Grosse-Kunstleve, R. W., Echols, N., Headd, J. J., Moriarty, N. W., Mustyakimov, M., Terwilliger, T. C., Urzhumtsev, A., Zwart, P. H., and Adams, P. D. (2012) Towards automated crystallographic structure refinement with phenix.refine. Acta Crystallogr., Sect. D: Biol. Crustallogr. 68, 352-367.

(24) Adams, P. D., Afonine, P. V., Bunkoczi, G., Chen, V. B., Davis, I. W., Echols, N., Headd, J. J., Hung, L.-W., Kapral, G. J., GrosseKunstleve, R. W., McCoy, A. J., Moriarty, N. W., Oeffner, R., Read, R. J., Richardson, D. C., Richardson, J. S., Terwilliger, T. C., and Zwart, P. H. (2010) PHENIX: a comprehensive Python-based system for macromolecular structure solution. Acta Crustallogr. Sect. D: Biol. Crustallogr. 66, 213-221.

(25) Chen, V. B., Arendall, W. B., III, Headd, J. J., Keedy, D. A., Immormino, R. M., Kapral, G. J., Murray, L. W., Richardson, J. S., and Richardson, D. C. (2010) MolProbity: all-atom structure validation for macromolecular crystallography. Acta Crystallogr. Sect. D: Biol. Crystallogr. 66, 12-21.

(26) Emsley, P., Lohkamp, B., Scott, W. G., and Cowtan, K. (2010) Features and development of Coot. Acta Crystallogr., Sect. D: Biol. Crystallogr. 66, 486-501.

(27) (2015) PyMOL Molecular Graphics System, Version 1.8, Schrödinger, LLC.

(28) Zeldin, O. B., Gerstel, M., and Garman, E. F. (2013) RADDOSE-3D: time- and space-resolved modelling of dose in macromolecular crystallography. I. Appl. Crvstallogr. 46, 1225-1230.

(29) Massey, V. (1991) A simple method for the determination of redox potentials. In Flavins and Flavoproteins 1990 (Curti, B., Ronchi, S., and Zanetti, G., Eds.), pp 59-66, Walter de Gruyter \& Co, Berlin.

(30) Cammack, R., Rao, K. K., Hall, D. O., Moura, J. G., Xavier, A. V., Bruschi, M., Le Gall, J., Deville, A., and Gayda, J.-P. (1977) Spectroscopic studies of the oxidation-reduction properties of three forms of ferredoxin from Desulphovibrio gigas. Biochim. Biophys. Acta, Protein Struct. 490, 311-321.

(31) Landau, M., Mayrose, I., Rosenberg, Y., Glaser, F., Martz, E., Pupko, T., and Ben-Tal, N. (2005) ConSurf 2005: the projection of evolutionary conservation scores of residues on protein structures. Nucleic Acids Res. 33, W299-W302.

(32) Ashkenazy, H., Erez, E., Martz, E., Pupko, T., and Ben-Tal, N. (2010) ConSurf 2010: calculating evolutionary conservation in sequence and structure of proteins and nucleic acids. Nucleic Acids Res. 38, W529-W533.

(33) Glaser, F., Pupko, T., Paz, I., Bell, R. E., Bechor-Shental, D., Martz, E., and Ben-Tal, N. (2003) ConSurf: Identification of Functional Regions in Proteins by Surface-Mapping of Phylogenetic Information. Bioinformatics 19, 163-164.

(34) Celniker, G., Nimrod, G., Ashkenazy, H., Glaser, F., Martz, E., Mayrose, I., Pupko, T., and Ben-Tal, N. (2013) ConSurf: Using Evolutionary Data to Raise Testable Hypotheses about Protein Function. Isr. I. Chem. 53, 199-206.

(35) Waterhouse, A. M., Procter, J. B., Martin, D. M. A., Clamp, M., and Barton, G. J. (2009) Jalview Version 2-a multiple sequence 
alignment editor and analysis workbench. Bioinformatics 25, 11891191.

(36) Sievers, F., Wilm, A., Dineen, D., Gibson, T. J., Karplus, K., Li, W., Lopez, R., McWilliam, H., Remmert, M., Söding, J., Thompson, J. D., and Higgins, D. G. (2011) Fast, scalable generation of high-quality protein multiple sequence alignments using Clustal Omega. Mol. Syst. Biol. 7, 539.

(37) Holm, L., and Rosenström, P. (2010) Dali server: conservation mapping in 3D. Nucleic Acids Res. 38, W545-W549.

(38) Jurrus, E., Engel, D., Star, K., Monson, K., Brandi, J., Felberg, L. E., Brookes, D. H., Wilson, L., Chen, J., Liles, K., Chun, M., Li, P., Gohara, D. W., Dolinsky, T., Konecny, R., Koes, D. R., Nielsen, J. E., Head-Gordon, T., Geng, W., Krasny, R., Wei, G. W., Holst, M. J., McCammon, J. A., and Baker, N. A. (2018) Improvements to the APBS biomolecular solvation software suite. Protein Sci. 27, 112-128.

(39) Guex, N., Peitsch, M. C., and Schwede, T. (2009) Automated comparative protein structure modeling with SWISS-MODEL and Swiss-PdbViewer: A historical perspective. Electrophoresis 30, S162S173.

(40) Bertoni, M., Kiefer, F., Biasini, M., Bordoli, L., and Schwede, T. (2017) Modeling protein quaternary structure of homo- and heterooligomers beyond binary interactions by homology. Sci. Rep. 7, 10480.

(41) Bienert, S., Waterhouse, A., de Beer, Tjaart, A. P., Tauriello, G., Studer, G., Bordoli, L., and Schwede, T. (2017) The SWISS-MODEL Repository-new features and functionality. Nucleic Acids Res. 45, D313-D319.

(42) Biasini, M., Bienert, S., Waterhouse, A., Arnold, K., Studer, G., Schmidt, T., Kiefer, F., Cassarino, T. G., Bertoni, M., Bordoli, L., and Schwede, T. (2014) SWISS-MODEL: modelling protein tertiary and quaternary structure using evolutionary information. Nucleic Acids Res. 42, W252-W258.

(43) Benkert, P., Biasini, M., and Schwede, T. (2011) Toward the estimation of the absolute quality of individual protein structure models. Bioinformatics 27, 343-350.

(44) Bashir, Q., Scanu, S., and Ubbink, M. (2011) Dynamics in electron transfer protein complexes. FEBS I. 278, 1391-1400.

(45) Muraki, N., Seo, D., Shiba, T., Sakurai, T., and Kurisu, G. (2010) Asymmetric Dimeric Structure of Ferredoxin-NAD(P)+ Oxidoreductase from the Green Sulfur Bacterium Chlorobaculum tepidum: Implications for Binding Ferredoxin and $\mathrm{NADP}^{+}$. L. Mol. Biol. 401, 403-414.

(46) Hayward, S., and Berendsen, H. J. C. (1998) Systematic analysis of domain motions in proteins from conformational change: New results on citrate synthase and T4 lysozyme. Proteins: Struct., Funct., Genet. 30, 144-154.

(47) Lennon, B. W., Williams, C. H., and Ludwig, M. L. (2000) Twists in Catalysis: Alternating Conformations of Escherichia coli Thioredoxin Reductase. Science 289, 1190-1194.

(48) Waksman, G., Krishna, T. S. R., Williams, C. H., Jr, and Kuriyan, J. (1994) Crystal Structure of Escherichia coli Thioredoxin Reductase Refined at $2 \AA$ Resolution: Implication for a Large Conformational Change during Catalysis. L.Mol. Biol. 236, 800-816. (49) Mulrooney, S. B., and Williams, C. H. (1997) Evidence for two conformational states of thioredoxin reductase from Escherichia coli: use of intrinsic and extrinsic quenchers of flavin fluorescence as probes to observe domain rotation. Protein Sci. 6, 2188-2195.

(50) Moser, C. C., Anderson, J. L. R., and Dutton, P. L. (2010) Guidelines for tunneling in enzymes. Biochim. Biophys. Acta, Bioenerg. 1797, 1573-1586.

(51) Komori, H., Seo, D., Sakurai, T., and Higuchi, Y. (2010) Crystal structure analysis of Bacillus subtilis ferredoxin-NADP ${ }^{+}$ oxidoreductase and the structural basis for its substrate selectivity. Protein Sci. 19, 2279-2290.

(52) Schilder, J., and Ubbink, M. (2013) Formation of transient protein complexes. Curr. Opin. Struct. Biol. 23, 911-918.

(53) Kobayashi, K., Ehrlich, S. D., Albertini, A., Amati, G., Andersen, K. K., Arnaud, M., Asai, K., Ashikaga, S., Aymerich, S., Bessieres, P., Boland, F., Brignell, S. C., Bron, S., Bunai, K., Chapuis, J., Christiansen, L. C., Danchin, A., Débarbouillé, M., Dervyn, E.,
Deuerling, E., Devine, K., Devine, S. K., Dreesen, O., Errington, J., Fillinger, S., Foster, S. J., Fujita, Y., Galizzi, A., Gardan, R., Eschevins, C., Fukushima, T., Haga, K., Harwood, C. R., Hecker, M., Hosoya, D., Hullo, M. F., Kakeshita, H., Karamata, D., Kasahara, Y., Kawamura, F., Koga, K., Koski, P., Kuwana, R., Imamura, D., Ishimaru, M., Ishikawa, S., Ishio, I., Le Coq, D., Masson, A., Mauël, C., Meima, R., Mellado, R. P., Moir, A., Moriya, S., Nagakawa, E., Nanamiya, H., Nakai, S., Nygaard, P., Ogura, M., Ohanan, T., O’Reilly, M., O’Rourke, M., Pragai, Z., Pooley, H. M., Rapoport, G., Rawlins, J. P., Rivas, L. A., Rivolta, C., Sadaie, A., Sadaie, Y., Sarvas, M., Sato, T., Saxild, H. H., Scanlan, E., Schumann, W., Seegers, J. F. M. L., Sekiguchi, J., Sekowska, A., Séror, S. J., Simon, M., Stragier, P., Studer, R., Takamatsu, H., Tanaka, T., Takeuchi, M., Thomaides, H. B., Vagner, V., van Dijl, J. M., Watabe, K., Wipat, A., Yamamoto, H., Yamamoto, M., Yamamoto, Y., Yamane, K., Yata, K., Yoshida, K., Yoshikawa, H., Zuber, U., and Ogasawara, N. (2003) Essential Bacillus subtilis genes. Proc. Natl. Acad. Sci. U. S. A. 100, 4678-4683. 